\title{
WiMAX Interference to MB-OFDM UWB Systems
}

\author{
Chris Snow, Lutz Lampe, and Robert Schober \\ Department of Electrical \& Computer Engineering, University of British Columbia, Vancouver, Canada
}

\begin{abstract}
This paper presents an analysis of the performance of the Multiband Orthogonal Frequency Division Multiplexing (MB-OFDM) system for Ultra Wideband (UWB) communication in the presence of interference from a single-carrier IEEE 802.16 WiMAX system operating in the $3.5 \mathrm{GHz}$ band. The intent of our analysis is to provide theoretical justification for the commonly-used bandlimited Gaussian noise model for this type of interference. We present an exact analysis of the uncoded bit error rate (BER) of the MB-OFDM system, based on Laplace transform techniques. We also present a simple and relatively accurate Gaussian approximation for the WiMAX interference.
\end{abstract}

\section{INTRODUCTION}

In this paper, we consider the ECMA-368 Multiband Orthogonal Frequency Division Multiplexing (MB-OFDM) standard for high rate Ultra Wideband (UWB) wireless communication [1], [2] in the 3.1-10.6 GHz band. Because UWB systems in this band are operating as spectral underlay systems [3], they will unavoidably be impacted by the transmissions of incumbent systems. In this paper, we consider the WiMAX 802.16 system for wireless Metropolitan Area Networks, operating in the licensed 3.5 GHz band [4]. The WiMAX standard consists of both single-carrier (SC) and OFDM-based modulation schemes. In this paper, we focus exclusively the single-carrier version of the standard for use below $11 \mathrm{GHz}$, referred to as WiMAX-SCa [4, Section 8.2].

When WiMAX is deployed in the $3.5 \mathrm{GHz}$ band, it will be a source of interference for MB-OFDM systems also using this band. For this reason, there has recently been some interest in co-existence techniques between WiMAX and UWB systems [5], [6]. Recent work also examines the effects of single-carrier narrowband interference on system design in MB-OFDM systems [7]. In most previous work, narrowband interference is modelled as bandlimited additive Gaussian noise. However, it is not immediately clear that such a Gaussian assumption holds in the case of WiMAX$\mathrm{SCa}$ interference to MB-OFDM systems, especially due to the wide range of allowable WiMAX operating bandwidths. The question of the validity of this assumption motivates our work herein.

In this paper, we investigate the effect of a WiMAX system operating in the $3.5 \mathrm{GHz}$ band and causing interference to an MB-OFDM system. In particular, we provide an exact analysis of the effect of the WiMAX system on the bit error

Email: \{csnow, lampe, rschober\}@ece.ubc.ca. The completion of this research was made possible thanks to Bell Canada's support through its Bell University Laboratories R\&D program and the Natural Sciences and Engineering Research Council of Canada (Grant CRDPJ 320 552), and with the support of a Canada Graduate Scholarship. This research was enabled by WestGrid computing resources, funded in part by the Canada Foundation for Innovation, Alberta Innovation and Science, BC Advanced Education, and participating institutions. Equipment is provided by IBM, HP, and SGI. rate (BER) of the MB-OFDM system, based on Laplace transform techniques. We then compare the exact analysis with a Gaussian approximation for the WiMAX interference signal.

\section{SYSTEM MODEL}

In this section, we describe the signal models for the MBOFDM transmitter and receiver, and for the WiMAX interferer.

\section{A. MB-OFDM Signal Model}

The transmitted MB-OFDM signal is given by

$$
s_{\mathrm{m}}(t)=\sum_{q=-\infty}^{\infty} \sum_{k=0}^{N_{\mathrm{m}}-1} x_{k, q} \phi_{k}\left(t-q T_{\mathrm{m}}\right) e^{j 2 \pi f_{\mathrm{m}} t},
$$

where $N_{\mathrm{m}}, T_{\mathrm{m}}$, and $f_{\mathrm{m}}$ are the number of subcarriers, the OFDM symbol duration, and the carrier frequency, respectively, cf. [1]. The transmitted Quaternary Phase Shift Keying (QPSK) symbols are denoted by $x_{k, q}$, where $k$ and $q$ represent the subcarrier index and the MB-OFDM symbol index, respectively. The basis function for subcarrier $k$ is given by

$$
\phi_{k}(t)=\left\{\begin{array}{ll}
\frac{1}{\sqrt{D_{\mathrm{m}}}} e^{j 2 \pi Q_{\mathrm{m}} k\left(t-C_{\mathrm{m}}\right)} & \text { if } t \in\left[0, T_{\mathrm{m}}\right] \\
0 & \text { otherwise }
\end{array},\right.
$$

where $C_{\mathrm{m}}, D_{\mathrm{m}}=T_{\mathrm{m}}-C_{\mathrm{m}}, W_{\mathrm{m}}$, and $Q_{\mathrm{m}}=W_{\mathrm{m}} / N_{\mathrm{m}}$ are the durations of the prefix and the data-carrying part of the OFDM symbol, the bandwidth of transmission, and the bandwidth per subcarrier, respectively, cf. [1]. While the MB-OFDM standard incorporates convolutional coding for error correction, we focus on uncoded modulation in this work in order to simplify the analysis. Ignoring the coding also allows us to isolate the contribution of the interference to the BER degradation, and to more clearly study possible approximations for the interference signal.

\section{B. WiMAX Signal Model}

The WiMAX transmitted signal is given by

$$
s_{\mathrm{n}}(t)=\sum_{\ell=-\infty}^{\infty} z_{\ell} p\left(t-\ell T_{\mathrm{n}}\right) e^{j 2 \pi f_{\mathrm{n}} t},
$$

where the modulated symbols are denoted by $z_{\ell}, f_{\mathrm{n}}$ and $T_{\mathrm{n}}$ are the WiMAX-SCa carrier frequency and symbol period, respectively, and $p(t)$ denotes the square-root raised cosine pulse shaping filter with rolloff factor 0.25 . The WiMAX standard specifies Binary Phase Shift Keying (BPSK), QPSK, 16-QAM (Quadrature Amplitude Modulation), 64-QAM, and 256-QAM modulation schemes [4]. For sake of space, we consider only BPSK and QPSK in this work, but note that similar analysis can be performed for the QAM schemes and similar results will be observed. 


\section{Channel Models and Receiver Processing}

The MB-OFDM signal passes through a channel with impulse response $h(t)$. Due to the relatively small WiMAXSCa bandwidth, and because of space limitations, we consider a single-tap WiMAX channel with amplitude $A$ and phase offset $\alpha$ uniformly distributed on $[0,2 \pi)$. The received signal, after downconversion to baseband and assuming that the interference signal lies in the band of interest, is given by

$$
r(t)=\left[s_{\mathrm{m}}(t) \otimes h(t)\right] e^{-j 2 \pi f_{\mathrm{m}} t}+i(t)+n(t),
$$

where $\otimes$ denotes the convolution operator, $n(t)$ is the additive white Gaussian noise (AWGN), and

$$
i(t)=A e^{j \alpha} s_{\mathrm{n}}(t-\tau) e^{-j 2 \pi f_{\mathrm{m}} t},
$$

where $\tau$ denotes the timing offset of the WiMAX signal, which is uniformly distributed on $\left[0, T_{\mathrm{n}}\right]$. For future reference, $\Delta \triangleq$ $f_{\mathrm{n}}-f_{\mathrm{m}}$ is the separation between the carrier frequencies of the two systems.

The baseband processing consists of a filterbank matched to $\phi_{k}(t)$ over $\left[C_{\mathrm{m}}, T_{\mathrm{m}}\right]$, and for subcarrier $k$ is given by

$$
\psi_{k}(t)=\left\{\begin{array}{ll}
\phi_{k}^{*}\left(T_{\mathrm{m}}-t\right) & \text { if } t \in\left[0, T_{\mathrm{m}}-C_{\mathrm{m}}\right] \\
0 & \text { else }
\end{array} .\right.
$$

Without loss of generality, MB-OFDM symbol index $q=0$, and the statistic for subcarrier $k$ is given by

$$
r_{k}=\left.\left(r(t) \otimes \psi_{k}(t)\right)\right|_{t=T_{\mathrm{m}}}=\tilde{y}_{k}+\tilde{i}_{k}+\tilde{n}_{k} .
$$

We note that, since the basis functions $\phi_{k}(t)$ are orthogonal, $\tilde{y}_{k}=G_{k} x_{k}$, where $G_{k} \triangleq g_{k} e^{j \eta_{k}}$ denotes the frequencydomain channel gain of subcarrier $k$ with magnitude $g_{k}$ and phase $\eta_{k}$, which is the sample of the Fourier transform of $h(t)$ at frequency $\left(f_{\mathrm{m}}+k Q_{\mathrm{m}}\right)$. The terms $\tilde{n}_{k}$ are complex AWGN variables.

The interference term can be expressed as

$$
\tilde{i}_{k}=\int_{C_{\mathrm{m}}}^{T_{\mathrm{m}}} i(t) \phi_{k}^{*}(t) \mathrm{d} t=A e^{j \alpha} \sum_{\ell=-\infty}^{\infty} z_{\ell} \beta_{k, \ell},
$$

where

$$
\beta_{k, \ell}=\int_{C_{\mathrm{m}}}^{T_{\mathrm{m}}} p\left(t-\ell T_{\mathrm{n}}-\tau\right) \phi_{k}^{*}(t) e^{j 2 \pi \Delta t} \mathrm{~d} t .
$$

\section{PERformance AnAlysis}

In this section, we provide an analysis of the BER for MBOFDM in the presence of WiMAX interference.

\section{A. BER Analysis with In-Band Interferer}

We start by noting that MB-OFDM employs QPSK modulation, which can also be considered equivalently as two independent BPSK modulations. As such, and noting that both $\tilde{i}_{k}$ and $\tilde{n}_{k}$ are rotationally symmetric, we can simplify our analysis by considering $x_{k, \ell}$ as BPSK symbols in the real plane and noting that the QPSK performance will be identical.
We can form the decision variable for subcarrier $k$ as

$$
\begin{aligned}
\Re\left\{e^{-j \eta_{k}} r_{k}\right\} & =\Re\left\{e^{-j \eta_{k}} \tilde{y}_{k}\right\}+\Re\left\{e^{-j \eta_{k}} \tilde{i}_{k}\right\}+\Re\left\{e^{-j \eta_{k}} \tilde{n}_{k}\right\} \\
& \triangleq y_{k}+i_{k}+n_{k},
\end{aligned}
$$

where $\Re\{\cdot\}$ denotes the real part of a complex number. Since we have assumed BPSK transmission, $y_{k} \triangleq \Re\left\{e^{-j \eta_{k}} \tilde{y}_{k}\right\}=$ $g_{k} x_{k}$, while $n_{k} \triangleq \Re\left\{e^{-j \eta_{k}} \tilde{n}_{k}\right\}$ are AWGN variables and $i_{k} \triangleq$ $\Re\left\{e^{-j \eta_{k}} \tilde{i}_{k}\right\}$ is given by

$$
i_{k}=A \Re\left\{e^{j\left(\alpha-\eta_{k}\right)} \sum_{\ell=-\infty}^{\infty} z_{\ell} \beta_{k, \ell}\right\} .
$$

For future reference, we define the signal-to-noise ratio (SNR) as

$$
\mathrm{SNR} \triangleq \frac{\mathbb{E}\left\{y_{k}^{2}\right\}}{\mathbb{E}\left\{2 n_{k}^{2}\right\}}=\frac{\mathbb{E}\left\{g_{k}^{2}\right\}}{2 \sigma_{n}^{2}},
$$

where $\sigma_{n}^{2}=\mathbb{E}\left\{n_{k}^{2}\right\}$ is the variance of $n_{k}$ (which is independent of $k$ ), and $\mathbb{E}\{\cdot\}$ denotes expectation. For subcarrier $k$, the signal-to-interference ratio $\left(\mathrm{SIR}_{k}\right)$ is given by

$$
\mathrm{SIR}_{k} \triangleq \frac{\mathbb{E}\left\{y_{k}^{2}\right\}}{\mathbb{E}\left\{2 i_{k}^{2}\right\}}=\frac{\mathbb{E}\left\{g_{k}^{2}\right\}}{2 \mathbb{E}\left\{A^{2}\right\} \sigma_{i, k}^{2}},
$$

where we have separated $\mathbb{E}\left\{A^{2}\right\}$ from $\sigma_{i, k}^{2}$ in order to account for possible random $A$, cf. Section III-B, and $\sigma_{i, k}^{2}$ is given by

$$
\sigma_{i, k}^{2}=\sum_{\ell=-\infty}^{\infty}\left|z_{\ell}\right|^{2}\left|\beta_{k, \ell}\right|^{2}=\sum_{\ell=-\infty}^{\infty}\left|\beta_{k, \ell}\right|^{2},
$$

since $\left|z_{\ell}\right|^{2}=1$. Given the MB-OFDM system hops over three bands, but that the interference power in two of the bands is zero, the overall average SIR is given by

$$
\mathrm{SIR} \triangleq \mathbb{E}\left\{g_{k}^{2}\right\} \cdot\left(\frac{2 \mathbb{E}\left\{A^{2}\right\}}{3 N_{\mathrm{m}}} \sum_{k=0}^{N_{\mathrm{m}}-1} \sigma_{i, k}^{2}\right)^{-1} .
$$

The symbols $x_{k}$ are equiprobable \pm 1 and $i_{k}$ and $n_{k}$ are zero mean and symmetric. Using properties of the Laplace transform [8], the probability of error for subcarrier $k$ is given by

$$
\begin{aligned}
P_{e, k} & =\operatorname{Prob}\left\{\left(i_{k}+n_{k}\right)<-g_{k}\right\}=\int_{-\infty}^{-g_{k}} p_{i_{k}+n_{k}}(x) \mathrm{d} x \\
& =\frac{1}{2 \pi j} \int_{c-j \infty}^{c+j \infty} \Phi_{i_{k}+n_{k}}(s) e^{-g_{k} s} \frac{\mathrm{d} s}{s}
\end{aligned}
$$

where $p_{i_{k}+n_{k}}(x)$ and $\Phi_{i_{k}+n_{k}}(s) \triangleq \mathbb{E}\left\{e^{-s\left(i_{k}+n_{k}\right)}\right\}$ denote the probability density function (pdf) of $\left(i_{k}+n_{k}\right)$ and its Laplace transform, respectively, and $c$ is a suitably chosen real value (discussed below). Due to the independence of $i_{k}$ and $n_{k}$

$$
\Phi_{i_{k}+n_{k}}(s)=\Phi_{i_{k}}(s) \Phi_{n_{k}}(s),
$$

and since $n_{k}$ is Gaussian, its Laplace transform is $\Phi_{n_{k}}(s)=$ $\exp \left(s^{2} \sigma_{n_{k}}^{2} / 2\right)$ [9]. We are left with the determination of $\Phi_{i_{k}}(s)$. We begin by considering the conditional Laplace 
transform $\Phi_{i_{k} \mid \tau, \alpha}(s)=\mathbb{E}\left\{e^{-s i_{k}} \mid \tau, \alpha\right\}$, which (since $z_{\ell}$ are independent) is given by

$$
\Phi_{i_{k} \mid \tau, \alpha}(s)=\prod_{\ell=-\infty}^{\infty} \mathbb{E}\left\{\exp \left(-s \Re\left\{A e^{j\left(\alpha-\eta_{k}\right)} z_{\ell} \beta_{k, \ell}\right\}\right)\right\}
$$

We now consider two choices of modulation scheme for the symbols $z_{\ell}$ of the WiMAX system:

1) BPSK WiMAX: We arrive at

$$
\Phi_{i_{k} \mid \tau, \alpha}(s)=\prod_{\ell=-\infty}^{\infty} \cosh \left(s \Re\left\{A e^{j\left(\alpha-\eta_{k}\right)} \beta_{k, \ell}\right\}\right) .
$$

2) QPSK WiMAX: We arrive at

$$
\begin{array}{r}
\Phi_{i_{k} \mid \tau, \alpha}(s)=\prod_{\ell=-\infty}^{\infty} \cosh \left(s \Re\left\{A e^{j\left(\alpha-\eta_{k}\right)} \beta_{k, \ell}\right\}\right) \times \\
\cosh \left(s \Im\left\{A e^{j\left(\alpha-\eta_{k}\right)} \beta_{k, \ell}\right\}\right),
\end{array}
$$

where $\Im(\cdot)$ denotes the imaginary part of a complex number.

We let $\alpha^{\prime}=\alpha-\eta_{k}$, and note that it is uniformly distributed on $[0,2 \pi)$. By integrating over the distributions of $\alpha^{\prime}$ and $\tau$, we obtain the Laplace transform $\Phi_{i_{k}}(s)$ as

$$
\Phi_{i_{k}}(s)=\frac{1}{2 \pi T_{\mathrm{n}}} \int_{0}^{T_{\mathrm{n}}} \int_{0}^{2 \pi} \Phi_{i_{k} \mid \tau, \alpha^{\prime}}(s) \mathrm{d} \alpha^{\prime} \mathrm{d} \tau .
$$

We can now determine the probability of error for subcarrier $k$, given by (15). Unfortunately, (15) does not have a closedform solution and we must resort to numerical evaluation. This can be done efficiently via the Gauss-Chebyshev quadrature rule [8]

$$
P_{e, k} \approx \frac{1}{K} \sum_{\nu=1}^{K / 2}\left(\Re\left\{\theta_{k}\left(c s_{\nu}\right)\right\}+\xi_{\nu} \Im\left\{\theta_{k}\left(c s_{\nu}\right)\right\}\right)
$$

where $\theta_{k}(\zeta) \triangleq \Phi_{i_{k}+n_{k}}(\zeta) e^{-g_{k} \zeta}, s_{\nu} \triangleq 1+j \xi_{\nu}, \xi_{\nu} \triangleq \tan ([2 \nu-$ $1] \pi /[2 K])$, and $K$ is a sufficiently large integer. We have found a good choice is $K=200$ for the computations in Section IV. In general, the real-valued parameter $c$ should be chosen to minimize $\theta_{k}(c)$. We have found that a simpler yet suitable choice of $c$ is the value which minimizes $\left.\theta_{k}(c)\right|_{\tau=0, \alpha=0}$, which can very quickly be determined using standard numerical techniques.

We now present an approximation for the BER in (15). Assuming that the interference signal at subcarrier $k$ with power $A^{2} \sigma_{i, k}^{2}$ can be modelled as an additional zero-mean Gaussian noise signal with variance $A^{2} \sigma_{i, k}^{2}$, where $\sigma_{i, k}^{2}$ is defined in (13), the effective noise power is given by $\sigma_{e, k}^{2,}=$ $\sigma_{n}^{2}+A^{2} \sigma_{i, k}^{2}$, and the BER for subcarrier $k$ is given by

$$
P_{a, k}=Q\left(\sqrt{g_{k}^{2} / \sigma_{e, k}^{2}}\right)
$$

where $Q(\cdot)$ is the Gaussian-Q function.

\section{B. Overall BER Analysis}

We first note that, when the MB-OFDM system is transmitting in a band where WiMAX interference is not present, the $\mathrm{BER}$ is given by

$$
P_{n, k}=Q\left(\sqrt{g_{k}^{2} / \sigma_{n}^{2}}\right) .
$$

In the general case, $A$ and $g_{k}$ are distributed according to probability density functions $p_{A}(A)$ and $p_{g_{k}}\left(g_{k}\right)$, respectively. In order to obtain the overall average BER in the presence of fading, we average (15), (19), and (20) over these densities.

We first consider (15), and take first the expectation over $g_{k}$

$$
\mathbb{E}_{g_{k}}\left\{P_{e, k}\right\}=\frac{1}{2 \pi j} \int_{c-j \infty}^{c+j \infty} \Phi_{i_{k}+n_{k}}(s) \mathbb{E}_{g_{k}}\left\{e^{-g_{k} s}\right\} \frac{\mathrm{d} s}{s},
$$

where we note $\Phi_{g_{k}}(s) \triangleq \mathbb{E}_{g_{k}}\left\{e^{-g_{k} s}\right\}$ is the Laplace transform of the pdf of $g_{k}$. Therefore (21) can again be evaluated using the Gauss-Chebyshev quadrature rule [8], cf. (18). The average exact BER in the presence of in-band interference is then given by

$$
\bar{P}_{e, k}=\int_{0}^{\infty} \frac{1}{2 \pi j} \int_{c-j \infty}^{c+j \infty} \Phi_{i_{k}+n_{k}}(s) \Phi_{g_{k}}(s) \frac{\mathrm{d} s}{s} p_{A}(A) \mathrm{d} A .
$$

We turn to the consideration of (19). We first take $\mathbb{E}_{g_{k}}\left\{P_{a, k}\right\}$, which, by using an alternative form of the Qfunction [9], can be written as

$$
\mathbb{E}_{g_{k}}\left\{P_{a, k}\right\}=\frac{1}{\pi} \int_{0}^{\pi / 2} M_{\gamma_{k}}\left(\frac{-1}{2\left(\sigma_{n}^{2}+A^{2} \sigma_{i, k}^{2}\right) \sin ^{2} \lambda}\right) \mathrm{d} \lambda,
$$

where $\gamma_{k}=g_{k}^{2}$, and $M_{\gamma_{k}}(s)=\mathbb{E}\left\{e^{s \gamma_{k}}\right\}$ is the moment generating function of $\gamma_{k}$ [9]. We can then express the average approximate BER in the presence of in-band interference as

$\bar{P}_{a, k}=\frac{1}{\pi} \int_{0}^{\infty} \int_{0}^{\pi / 2} M_{\gamma_{k}}\left(\frac{-1}{2\left(\sigma_{n}^{2}+A^{2} \sigma_{i, k}^{2}\right) \sin ^{2} \lambda}\right) \mathrm{d} \lambda p_{A}(A) \mathrm{d} A$

Using similar techniques as with (23), we can express the average BER without interference as [9]

$$
\bar{P}_{n, k}=\frac{1}{\pi} \int_{0}^{\pi / 2} M_{\gamma_{k}}\left(\frac{-1}{2 \sigma_{n}^{2} \sin ^{2} \lambda}\right) \mathrm{d} \lambda .
$$

For first generation devices, the MB-OFDM hops over three bands with equal average usage, and the WiMAX system of interest is found in the first band. Thus, the overall BER is

$$
\bar{P}=\frac{1}{3}\left(\frac{1}{N_{\mathrm{m}}} \sum_{k=0}^{N_{\mathrm{m}}-1} \bar{P}_{X, k}\right)+\frac{2}{3}\left(\frac{1}{N_{\mathrm{m}}} \sum_{k=0}^{N_{\mathrm{m}}-1} \bar{P}_{n, k}\right),
$$

with $X \in\{e, a\}$ depending on whether (22) or (24) is used. If $g_{k}=1$ and $A=1$, i.e., if both the WiMAX and MB-OFDM signals are non-faded, we can simplify (26) by substituting (20) and (18) or (19). 


\section{RESULTS AND DISCUSSION}

In this section, we present numerical results illustrating the performance analysis methods presented in Section III. To focus more clearly on the effect of the interference, and due to space limitations, we concentrate on the case of $A=1$ and $g_{k}=1 \forall k$, i.e., the case of non-fading channels for both the MB-OFDM and WiMAX transmissions. The parameters of both systems are chosen from the standards [1], [4]. The results below have also been verified via simulations, which we have omitted due to space limitations.

We first consider WiMAX-SCa with BPSK modulation. In Figure 1 we plot the BER versus $10 \log _{10}$ (SIR) for different values of WiMAX-SCa bandwidth, for fixed $10 \log _{10}(\mathrm{SNR})=$ 10. We show both the exact analysis (lines) and the Gaussian approximation (markers). The Gaussian approximation is very accurate for small and large SIR, with some deviation at intermediate values of SIR. We can also see that the Gaussian approximation is worst for small values of WiMAX-SCa bandwidth, and improves as the bandwidth increases. This is due to the shorter symbol time of the wide bandwidth WiMAX-SCa signal, leading to a more pronounced averaging effect of interference during one MB-OFDM symbol duration. We also note that, for a fixed $10 \log _{10}(\mathrm{SIR})$, the BER tends to decrease as the interferer bandwidth increases. This is because the per-subcarrier interference power decreases as the bandwidth increases (since the average interference power is constant), and thus (since the BER decays exponentially with increasing $\mathrm{SIR}_{k}$ ) the values of $P_{e, k}$ also decrease with increasing interference bandwidth.

In Figure 2 we consider QPSK WiMAX modulation, and plot the BER versus $10 \log _{10}(\mathrm{SIR})$ for different values of WiMAX bandwidth. We can see the Gaussian approximation is improved in comparison with Figure 1, due to the increased randomness of the four-phase QPSK signal. We note that similar trends have been observed for OFDM interference signals [10]. We expect the Gaussian approximation to continue to improve for the higher-order QAM modulations.

In summary, we have obtained exact expressions for the uncoded BER of an MB-OFDM system in the presence of interference from a WiMAX-SCa system. We have seen that the Gaussian approximation is accurate for small and large values of SIR, and deviations at intermediate SIR tend to decrease with increasing WiMAX-SCa bandwidth and modulation order.

\section{REFERENCES}

[1] ECMA, "Standard ECMA-368: High Rate Ultra Wideband PHY and MAC Standard," Dec. 2005, [Online]: http://www.ecmainternational.org/publications/standards/Ecma-368.htm.

[2] A. Batra, et al., "Design of a Multiband OFDM System for Realistic UWB Channel Environments," IEEE Trans. Microwave Theory Tech., vol. 52, no. 9, pp. 2123-2138, Sept. 2004.

[3] Federal Communications Commission (FCC), "Revision of Part 15 of the Commissions Rules Regarding Ultra-Wideband Transmission Systems," First Report and Order, February 14, 2002.

[4] IEEE Std 802.16-2004, "Part 16: Air Interface for Fixed Broadband Wireless Access Systems," Oct. 2004.

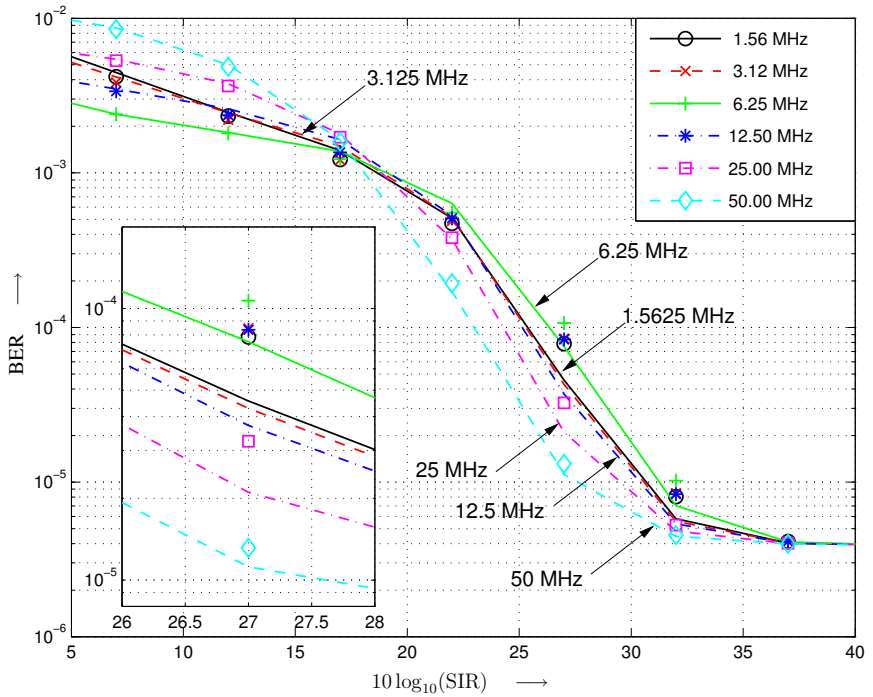

Fig. 1. BER versus $10 \log _{10}(\mathrm{SIR})$ for $10 \log _{10}(\mathrm{SNR})=10$ and various values of WiMAX-SCa bandwidth, with exact analysis (lines) and Gaussian approximation (markers). Inset: Zoomed version of same figure, showing difference between Gaussian and exact BER. BPSK WiMAX-SCa modulation.

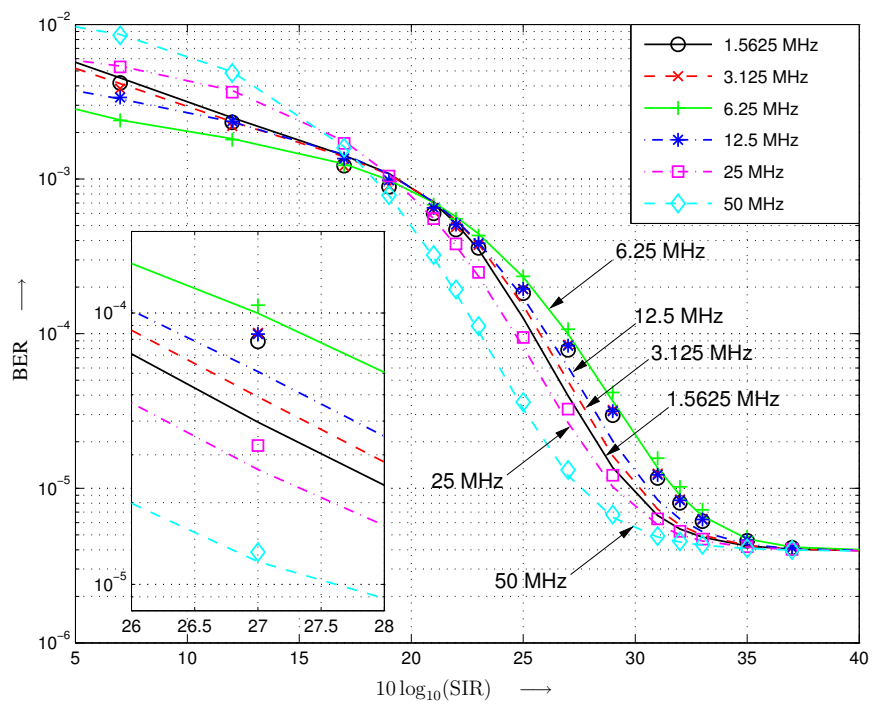

Fig. 2. BER versus $10 \log _{10}(\mathrm{SIR})$ for $10 \log _{10}(\mathrm{SNR})=10$ and various values of WiMAX-SCa bandwidth, with exact analysis (lines) and Gaussian approximation (markers). Inset: Zoomed version of same figure, showing difference between Gaussian and exact BER. QPSK WiMAX-SCa modulation.

[5] V. Somayazulu, J. Foerster, and R. Roberts, "Detect and Avoid (DAA) Mechanisms for UWB Interference Mitigation," in Proc. IEEE Intl. Conf. on Ultra-Wideband, Waltham, MA, USA, Sept. 2006, pp. 513-518.

[6] A. Durantini, et al., "Performance Evaluation of Detect and Avoid Procedures for Improving UWB Coexistence with UMTS and WiMAX systems," in Proc. IEEE Intl. Conf. on Ultra-Wideband, Waltham, MA, USA, Sept. 2006, pp. 501-506.

[7] K. Shi, et al., "Impacts of Narrowband Interference on OFDM-UWB Receivers: Analysis and Mitigation," IEEE Trans. Signal Processing, vol. 55, no. 3, pp. 1118-1128, Mar. 2007.

[8] E. Biglieri, et al., "Computing Error Probabilities over Fading Channels: a Unified Approach," European Transactions on Telecommunications, vol. 9, pp. 15-25, January/February 1998.

[9] M. K. Simon and M.-S. Alouini, Digital Communication over Fading Channels, 2nd ed. Wiley, 2005.

[10] B. Hu and N. C. Beaulieu, "Performance of an Ultra-Wideband Communication System in the Presence of Narrowband BPSK- and QPSKModulated OFDM Interference," IEEE Trans. Commun., vol. 54, no. 10, pp. 1720-1724, Oct. 2006. 